\title{
A SIMPLE CCD-SYSTEM FOR SECONDARY ALIGNMENT OF THE SPECTRUM-UV SPACE TELESCOPE.
}

\author{
L. V. Didkovsky, N. V. Steshenko, P. I. Borzyak and A. I. Dolgushin \\ Crimean Astrophysical Observatory
}

\begin{abstract}
A CCD-system is proposed to maintain the alignment of an optical system of a large space telescope during ground tests and after launch. The system is able to detect alignment errors resulting from displacements of the main and secondary mirrors of the telescope and also from the tilts of these mirrors. Computer simulations have been performed to determine the sensitivity of the system to the displacements and/or the tilts. Different sources of errors have been also explored on a working stand; and the main requirements to the elements of the system have been determined. The system is to be installed on the space telescope "Spectrum-UV". It can be also used in ground-based telescopes in a quasi-real time mode.
\end{abstract}

\section{INTRODUCTION}

A new space Ritchey-Chrétien telescope "Spectrum-UV", which is currently being designed, will be equipped with spectrometers installed at a significant distance $(50 \mathrm{~mm})$ from the center of the field of view. Therefore, any displacement or tilt of the secondary mirror (SM) may strongly affect the quality of the image in the spectrometers, even if the disturbances do not change the quality of the image significantly in the center of the field of view. Our task was to develop a system which would maintain the alignment of an optical system of a telescope, the Secondary Alignment System (SAS).

\section{2, INSTRUMENTATION}

The SAS system must detect different breaches of alignment and separate those connected with decentering of the SM from breaches caused by tilts of the SM. The optical scheme of the SAS (Fig. 1.) consists of two measuring channels. The first channel includes the source of light $\mathrm{S} 1$, placed on the main mirror (MM), the flat mirrors M1 and M3 manufactured right on the $\mathrm{SM}$ as one of its parts, and the spherical mirror M4, which is manufactured as a part of the MM and serves for focusing the optical beam into the CCD. This channel is practically insensitive to any transversal motions of the SM. It detects only tilts of the SM. The second measuring channel consists of the source of light S2, placed on the MM, the spherical mirror M2 manufactured as a part of the SM, and the same CCD. The second channel is sensitive to both the displacements and the tilts of the SM. We have used a CCD camera with 256 x 288 array and 8-bit ADC. Using the CCD as a sensor, we have been able to determine the position of the center of the image with high precision in a rather wide range of displacements. It seems also to be important that the positions of the optical beams can be stored in the form of the $\mathrm{X}, \mathrm{Y}$ coordinates of the beam rather than as electrical levels, that makes maintaining of the alignment more reliable. 

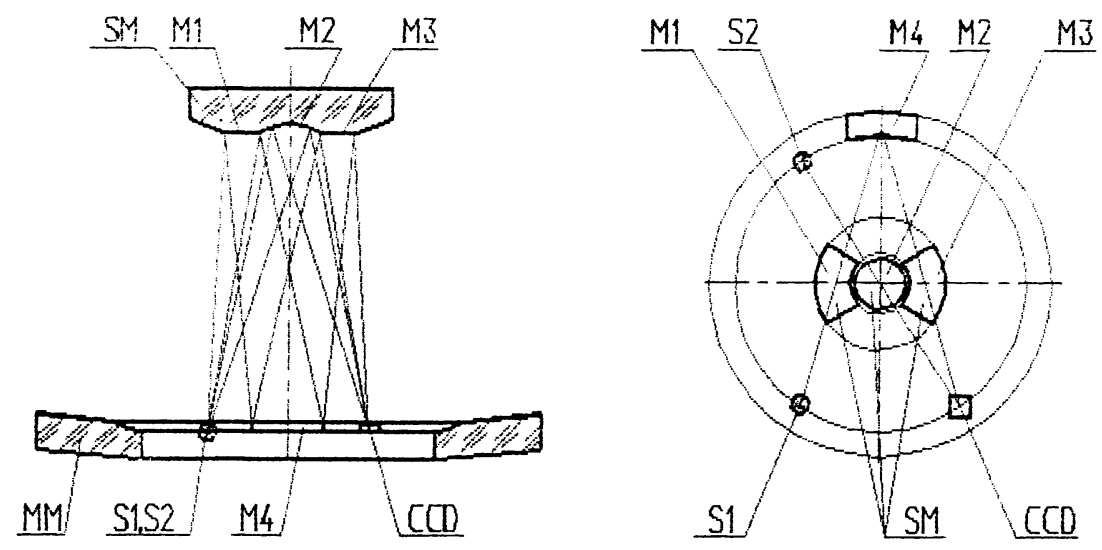

Fig. 1. Optical scheme of the Secondary Alignment System.

\section{MODELING AND TESTING}

We have performed computer simulations of the optical scheme of the SAS studying responses of the system in the whole possible range of the tilts and displacements of the SM. All technical tolerances for the components of the system have been taken into account; and all necessary requirements for manufacturing have been set up. We have also analyzed the main factors which affects precision of the determination of the alignment breaches. The sensitivity of the system, which is defined as minimal measurable displacement of a lightspot on the CCD, has to be about $10 \mu \mathrm{m}$ to provide the required quality of image. However, our laboratory measurements have demonstrated that the real sensitivity of the system is significantly higher even under ground conditions.

\section{CONCLUSION}

We have developed a Secondary Alignment System for the space telescope "Spectrum UV", which will enable us to follow every breach in co-axiality between the main and the secondary mirrors with settled precision. The information about any alignment breaches will be used during ground-based testing of the telescope. The system gives also ability to restore the ground-made alignment and to reach the required image quality for the whole width of the field of view of the telescope after its launch. 